\title{
A Service Integration Platform for Collaborative Networks
}

\author{
A. Luis Osório ${ }^{1,2}$, Hamideh Afsarmanesh ${ }^{2}$, Luis M. Camarinha-Matos ${ }^{3}$ \\ ${ }^{1}$ Instituto Superior de Engenharia de Lisboa, Lisboa Portugal (aosorio@isel.pt) \\ ${ }^{2}$ University of Amsterdam, Amsterdam Netherlands (h.afsarmanesh@uva.nl) \\ ${ }^{3}$ Faculdade de Ciências e Tecnologia, Universidade Nova de Lisboa, Portugal (cam@uninova.pt)
}

\begin{abstract}
Integrated manufacturing constitutes a complex system made of heterogeneous information and control subsystems. Those subsystems are not designed to the cooperation. Typically each subsystem automates specific processes, and establishes closed application domains, therefore it is very difficult to integrate it with other subsystems in order to respond to the needed process dynamics. Furthermore, to cope with ever growing market competition and demands, it is necessary for manufacturing/enterprise systems to increase their responsiveness based on up-to-date knowledge and in-time data gathered from the diverse information and control systems. These have created new challenges for manufacturing sector, and even bigger challenges for collaborative manufacturing. The growing complexity of the information and communication technologies when coping with innovative business services based on collaborative contributions from multiple stakeholders, requires novel and multidisciplinary approaches. Service orientation is a strategic approach to deal with such complexity, and various stakeholders' information systems. Services or more precisely the autonomous computational agents implementing the services, provide an architectural pattern able to cope with the needs of integrated and distributed collaborative solutions. This paper proposes a service-oriented framework, aiming to support a virtual organizations breeding environment that is the basis for establishing short or long term goal-oriented virtual organizations. The notion of integrated business services, where customers receive some value developed through the contribution from a network of companies is a key element.
\end{abstract}

Keywords: Collaborative Networks; Virtual Organizations; Service Oriented Computing; Service Oriented Architecture.

\section{Introduction}

There is an increasing need for integrated solutions in manufacturing which in turn requires a strategy to create integrated knowledge views grounded on contributions from the underlying manufacturing and information systems. This trend is not restricted only to manufacturing or enterprise domain, rather to many sectors and environments in need of collaborative networks, and where organizations need to experience new and novel (collaborative) business models. Focusing on the business models related to integrating business services, the existing information and communication infrastructures do not sufficiently support their required level of integration [13]. In fact, the existing information systems tend to follow proprietary technology on their information modeling and processing patterns. They are primarily developed to run as standalone systems, and automate a group of processes, without concerns for potential cooperation needs with other processes. Furthermore, since processes are based on hard-coded business logic, the existing information systems are closed and cannot easily evolve to answer new changes or requirements in their environment. This drawback is particularly identified in large scale and critical systems where the system operation and security are of paramount importance. In a closed or proprietary system, even when standards are adopted, there is always the system's original supplier, typically the vendor (developer/integrator), who keeps the responsibility to cope with adjusting to requirement changes, and providing the system's life cycle management. Nevertheless, the emerging complexities of the manufacturing/enterprise information systems require that subsystems can be easily integrated, hence requiring novel approaches for their support. A key approach strategy is to reduce dependencies at the single system level, and to make the integrated system as a composition of open subsystems (Figure 1). The difference between a closed system and an open system shall then be notices and argued, while adopting standards and aiming to present an open system/solution.

Existing closed complex systems are not able to evolve without being maintained by the original supplier of the integrated system. This creates contractual dependencies to the 


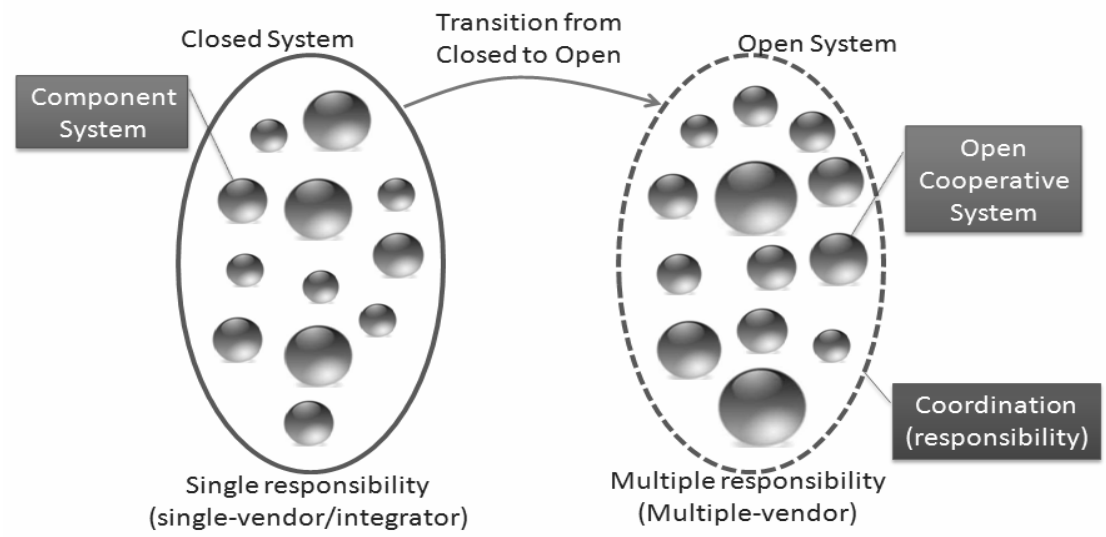

Figure 1. A transition from closed to open systems.

contracting organization(s), with a weak capacity to influence the evolution of such a system. The responsibilities which remain with the original supplier, as well as the risks involved if additional components from a different supplier are applied to the original system, are a main obstacle to reduce market dependencies. A novel framework and infrastructure needs to be introduced to support the development of open complex system, capable of integrating independently developed subsystems, supplied by different providers/vendors within competitive market place. An open research question is how to make the transition to an equivalent open complex system by maintaining an equivalent robustness and security. Existing market service oriented software suites establish technology dependencies making difficult a transition to open service-oriented enterprise architectures [8]. Trust and reputation mechanisms [20] are discussed and strategies are proposed to address open dynamic service-oriented environments where distributed computational systems (service systems) can cooperate to collaboratively develop business processes [14], also referred to as cross-organizational business process (CBP) [18]. Nevertheless, developing such complex distributed system of systems requires a novel framework, which can establish an open collaborative space.

Service oriented architecture (SOA) and service oriented computing (SOC) are concepts that have been developed both by research community and industry as innovative patterns to structure the growing complexity of computational infrastructures needed to cope with complex integrated solutions. One main motivation has been the risks of increased dependency of the complex integrated solutions on the proprietary approaches, which in turn make them too dependent on specific suppliers. From the engineering viewpoint, existing integrated solutions tend to follow a "one-of-a-kind" approach making them too expensive not only at the development phase but also along its overall life cycle. In line with service oriented architecture, an initiative such as the service component architecture (SCA) proposes a strategy to develop assemblies of distributed and heterogeneous autonomous components from the same or different providers. Another contribution comes from the open services gateway initiative (OSGi) that proposes a framework to add agility to the development of applications based on loosely coupled components. The proposed SCA framework is an interesting approach for combining distributed heterogeneous and autonomous computational entities into integrated solutions. Nevertheless the simple combination of heterogeneous and autonomous components originated from different suppliers is not enough to cope with the growing complexity and needs of collaborative networks. Especially when considering the complete life cycle - from, development, deployment, operations management and evolution.

While SOA and SOC tackle complexities in distributed systems, it is fundamental to establish a clear semantics for the concept of service [19]. The organization for the advancement of structured information standards (OASIS) suggests a holistic approach to SOA systems, as networks of a number of entities including service 
providers, individuals (acting at different roles), machines, suppliers, entities that directly or indirectly establish a system's behavior [9]. More conservative approaches point to an enterprise service bus (ESB) as a structured evolution from enterprise application integration (EAI), where message oriented middleware and data/information transformations play a major role, while they do not present significant innovations. Even when a service wrapper layer is proposed to transform legacy applications/systems into more normalized components, we can question whether this is the right strategy to cope with the requirements of more complex integrated systems.

As pointed out by the IEEE Transactions on Services Computing initiative, a necessary convergence of semantics around service oriented computing is expected to bring some clarity to the currently dispersed contributions. This new body of knowledge is organized around four main areas: i) services and service systems; ii) service technologies; iii) services consulting and delivery; and vi) services applications and management [22]. This and other complementary initiatives are being pushed by a class of new business services offering people new facilities, and involving contributions from different stakeholders, while making the underlying business arrangements transparent to clients. A paradigmatic example is the utilization of a unique business card (or business nomadic card) that can be used by an individual to pay for public transports, newspapers, street parking, and shopping in stores, based on a unique contract with an integrated service provider. These new collaborative business scenarios, where critical processes rely on a number of diverse information systems, require an innovative distributed computational infrastructure closer to the notion of service ecosystem or service park.

This paper proposes a strategy for creation of a service ecosystem integrating multicultural technical systems through declarative semantics (ontology) in order to make such ecosystem sufficiently agile to cope with the emerging collaborative business scenarios offering integrated value-added services to the customers. For instance, when considering integrated solutions for many different running computational service systems at a large complex building, a large shopping centre, a hospital or a public services building, there is also a need for an integrated management and supervision solution to support interoperation among these services. The needed solution must support and shall span over the basic subsystems, e.g. programmable logic controllers (PLC), video camera infrastructures with video server recorders (VSR), heating, ventilation, and air conditioning systems (HVAC), and many other specialized subsystems, which are provided by different technological stakeholders. The open question is how to model and effectively support a network of stakeholders, with offering a single integrated holistic approach to the end-users, while keeping the independence among the service providers.

\section{Balanced Approach for Integration of Business Services}

The notion of integrated business service is not an entirely new concept, as it can be perceived whenever business challenges require a multidisciplinary approach for service provision or the complexity and the need to reduce operational risks requires the participation of more than one company in a given business opportunity. Such services require a balanced approach considering the need for the participation of technology, process, and business expertise. The integrated business service can be linked to the collaborative network (CN) concept as it results from the contribution of heterogeneous and autonomous distributed entities (e.g. organizations, people, software agents) that collaborate to achieve common objectives through sharing their resources and capabilities and thus jointly creating new values [5], [23]. Integrated business services are much more efficiently grounded if they are established within a long-term business alliance that can serve as the breeding environment for formation of business-based collaborations; namely within a virtual organizations breeding environment (VBE) [1]. The concept of VBE has been proposed to guarantee a minimum collaboration preparedness state for its members, as a precondition to participate in collaborative business opportunities, i.e., as the ground for 
supporting the complete lifecycle of virtual enterprises/virtual organizations (VE/VO) [6].

Establishing an integrated business service (IBS) also requires an adequate preparedness state from its candidate stakeholders in line with the VO concept. From the ICT development point of view, the requirements identified in [2] for a collaborative network, namely: i) ICT-I reference framework; ii) ICT-I business models; iii) Security framework; and iv) ICT SOA-based infrastructure for collaboration, represent an important basis for the definition of a generic ICT infrastructure for integrated business services. Similar requirements come from other domains as the ePAL/BRAID roadmaps [7] on ICT and ageing.

At present some IT supplier companies tend to offer some integrated platforms in response to the increasing needs for integrated solutions. As identified in [11], this implies that then the complementary networked stakeholders shall negotiate long term service level agreements (SLA) with the IT provider company in order to jointly develop an IBS and establishing their collaborative business relations. Nevertheless, such IT suppliers typically tend to promote monolithic closed solutions, and find it very difficult to cope to the $\mathrm{CN}$ requirements of providing an open infrastructure. The open approach is even seen as a threat by some market players.

While most proposed research in the area of $\mathrm{CN}$ is centered on identifying the dynamics of business ecosystems involving producers, service providers, consumers, certification bodies, and regulators, with a focus on the business patterns, our research follows a complementary approach to the proposed integrated business service concept. In our approach IBS emerges from collaborative network ecosystems - VBEs - made of complementary autonomous stakeholders which possess their own heterogeneous information systems. Therefore, the discussion is centered on the networked integration perspective, i.e. an integration of contributions (services) linked to diverse IT systems, held by the participating stakeholders. As such, an integrated business service can be defined as a service provided by a network of stakeholders, which are transparent to the subscribing end-user, namely under the assumption that the service subscriber assumes only one contractual relation. In this way, an integrated business service establishes a virtual provider that might be operated and coordinated by a new organizational entity (a new company, a temporary legal entity, or an association where one participating company leads and supports the collaboration). Furthermore, the supporting persons and IT resources are spread over a network. Each participating stakeholder in such collaborative network has its own information system related to its specific organization domain. Organization domains (OD) establish a trusted execution context under a unified organization's intraenterprise information system with its associated collaboration layer (Figure 3).

Provision of an integrated business service requires an extended cooperative space beyond the intra-organization domain. Nevertheless, a key requirement is the need for some unified platform even considering that each participating organization has its own IT system relying on some specific architecture and technology bindings. Furthermore, each organization has its own specific business process framework(s) adapted to its internal process models. On the one hand it is advisable to promote a tight integration between intra organizations' processes and the collaborative processes, those contributing to the realization of the integrated business services. But on the other hand, establishment of strong dependencies among stakeholders when participating in the processes that support integrated business services is against the openness approach and is not desirable or even realistic in time. This situation points to the need for a strategy that can preserve organization's autonomy while coping with collaboration contractual agreements that underlie integrated business services. As an example, an evolution in one stakeholders' information system should not affect the behavior of the running processes and services participating in collaborative IBPs [17]. Our proposed strategy considers existence of a collaboration layer (CL) at every participating stakeholder, thus providing a separation of contexts between the domain of intra-enterprise information 
systems, and that of the network collaboration domain.

For instance, in an intelligent transportation network, an integrated business service can offer an innovative insurance model based on drivers' behavior, which can be identified through the data collected from an enhanced on-board unit, similar to the one nowadays used for tolling (on-board dedicated short range communication transponder). This gives an example of the need for the participation (cooperation) of several systems that can be managed by: a tolling service provider, multiple highway operators (multiple concessionaires) and an insurance company. When a vehicle crosses a tolling infrastructure, the needed information collected from vehicle bus (CAN bus) is sent to the tolling stakeholder in conjunction with the tolling transaction data. As such, within the same contract that was initially exclusively related to highway tolling, the above insurance company, in cooperation with the business integrated service provider, can now offer an innovative insurance fee calculation for customers. Therefore, the extracts (cost statements for customers) can be enhanced with the insurance payments when presented to subscribers, depending on the agreed payment periods (e.g. daily, weekly, monthly or yearly). The example of the Spanish insurance company Mapfre that has developed a pay-as-you-drive (PAYD) model, based on a specialized on-board system, is discussed in [21]. Another similar example is the integrated business service offered by the Portuguese Via-Verde company, which in the same contract covers both the tolling and car parking payments. It is a running example where collaboration among the involved organizations follows a specific IT infrastructure design and developed mechanisms [15], even though the information systems of some of the involved stakeholders has evolved to a service oriented culture.

A similar situation can be foreseen for the provision of care services to elderly, in which different entities e.g. care centers, health centers, social security, leisure centers, etc., can contribute to provide integrated services under a single contract. These examples suggest the need for a balanced approach where VBEs might need to involve members at different IT development stages, motivating the proposal of introducing the collaboration layer.

\section{Cooperative Open Service Infrastructure}

The integrated business services often require the involvement of a grid of diverse resources, including the computational services, persons interacting through multiple communication channels (human machine interaction channels), and devices (also represented by services), which are able to participate in different collaborative processes. Such resources need to all be represented as adaptive entities or components, embedding meta-information, and able to be managed in different lifecycle phases of the IBSs. In such an environment, collaborative processes can be provided, realizing integrated business services, through the composition of a hierarchy of processes, each one relying on the composition of other already defined simple or composite services.

In this framework the finest grained entity is a simple service seen as an autonomous computational entity, implemented in some technology (e.g. Web Services, JINI, WCF, Multi-agent framework, grid-services). Here, the entire computational logic, even if associated to monolithic systems such as a classic enterprise resource planning (ERP) application, is abstracted as services that are supported through wrappers, according the proposed framework. Motivated by the need to increase reutilization of computational logic, services can be organized in two main classes: i) system services, and ii) application services. The system services are those autonomous computational entities, possibly from different suppliers, that are part of the organization's open service bus. They contribute to establish the ICT culture of the organization, i.e. providing a kind of organization's signature. The planned strategy is to encourage (or force) new service suppliers to reutilize as much as possible the existing resources, instead of developing from scratch. As an example, a new application service for managing human resources (or simply to add a new service to the human resource management facilities) 
must use the already existing and documented identity management system service. Therefore, a system service is part of the organization's open service bus. On the other hand, an application service is not conditioned to offer services to other peer services and thus it is not part of the organization's open service bus.

The logical structure of an application or system service, while prepared to be involved in collaborative processes, involves four primary aspects:

1. A functional interface making available a number of operations under some predefined semantics (implicit or explicit semantics through the association of an ontology). A functional interface can include operations like add (op1, op1), multiply (op1, op1), as part of a calculator service.

2. Event infrastructure responsible to manage asynchronous relationships among services. Consider a subscription of an event signaling the subscriber each time a functional operation is called in the calculator;

3. Instrumentation port to integrate a monitoring infrastructure based on specialized diagnostic and prognostic services (agents);

4. Meta-data embedding the necessary parameters to support service client's adaptation. This can be done through implicit semantics, data structures and semantics resulting from a normalization process or adopting an ontology, thus offering dynamic adaptability to potential clients.
The events infrastructure makes it possible to subscribe to certain events, thus providing an asynchronous access to service relevant information. A client service can subscribe to relevant events directly from their originating services or can access the generated events through a tuple space (in this case shared by all other services in the collaboration network). The utilization of a tuple-space avoids the need for services to know the event ports for each event originating service.

The instrumentation port aims at establishing an open monitoring infrastructure based on coordinated monitoring agents which are specialized to guarantee the correct operation of the running services. This strategy is inspired by the existing monitoring infrastructures for communication systems, like the routers and switches. Network management applications can monitor system's behavior by accessing a management information base through the single network management protocol or using other open standards like the Java management extensions protocol and the corresponding managed Beans.

The associated meta-data is organized under two main groups: i) the information related to the infrastructure, and ii) the information related to the collaboration in the context of a collaborative network (Figure 2). Both service information (meta-data) groups are organized in two sub groups: i) the acquaintance information holding the knowledge required by potential service clients and market-place

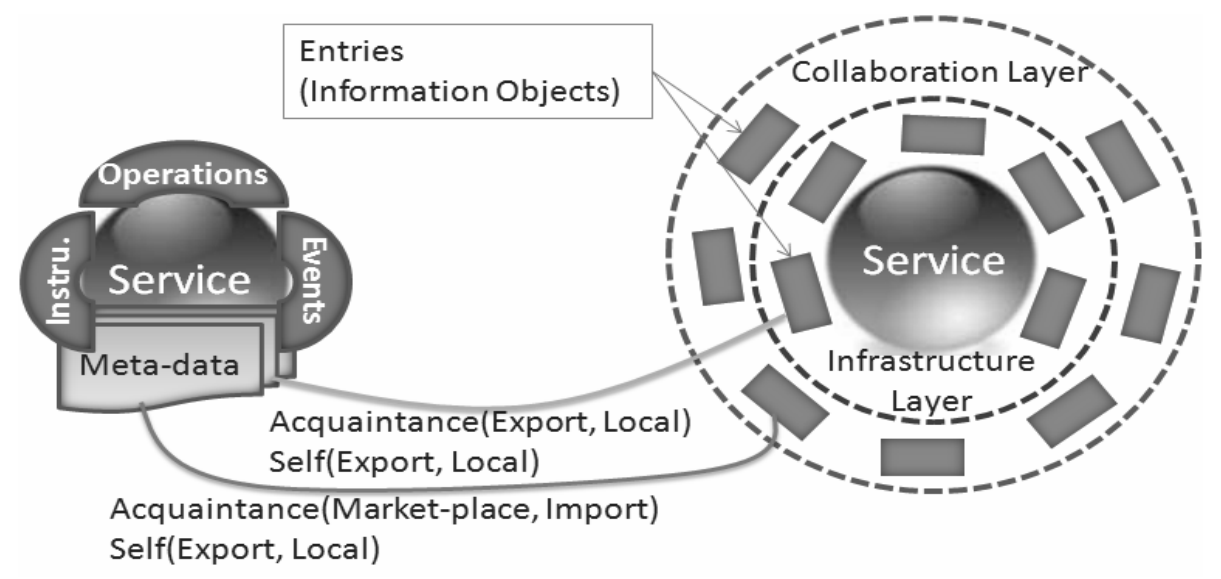

Figure 2. Model of a service in a collaborative network.

The functional interface establishes a number of operations to be accessed by other services (clients) according their functional needs. to discover and select decision support, and ii) the self information addressing the infrastructure configuration requirements. 
The open service framework (OSF) for collaboration among organizations is made of cooperative enabled services (CES) which are available from a market place. One important difference from existing approaches namely the cooperative processes defined by business process modeling notation (BPMN) where collaboration is associated to exchange of messages among organizations, is that the proposed framework considers the CES as public services available to participate in collaborative processes. A collaborative process is enacted on a collaborative network infrastructure, established by the proposed OSF. Furthermore, any service in one organization can access a CES in any other organization within the same collaborative network (CN). This means that all CESs, provided by different service providers within a $\mathrm{CN}$, establish a basis for design of new specialized services, which can be developed and deployed as compositions of the base services available in the network. If the collaboration layer of a service has no defined information, it is considered a private service and as such it is not visible at the collaborative network level.

In such a service space at least one service must play the role of service repository, which might be implemented as an LDAP, JINI/Reggie, UDDI, Active Directory or other directory service. To cooperate with other services, a service first looks up at the repository and selects the needed subset of services that are needed in order to generate a cooperative integrated solution (Figure 2).

\section{Open Collaborative Business Framework}

Most existing ICT solutions are so far developed without embedding any capabilities to support their potential future integration with other solutions. This means that existing systems/applications are developed in response to specific functional and/or other requirements, following a software development process considering the completeness of the developed solution against the expected results. Even when solutions are based on open source, their considered criteria and advantage is on an eventual reduction of the total costs by significant reduction in their development- time and complexity as well as the run-time costs. Nevertheless, the costs associated to the extra development and configuration required for open source components may generate expensive solutions.

What is expected to contribute to the reduction of the total cost of ownership of integrated solutions is the approach of grounding the development of complex integrated solutions on a multi-vendor computational autonomous market place. This requires a new approach to service development and establishing a high level language able to abstract a multitechnology and multi-supplier computational infrastructure. In this line, the enterprise generation language (EGL) from IBM [10] is an example of the market recognition of the fact that something new is needed to cope with the complexity associated to the development of integrated solutions made of an evolving diverse set of resources, e.g. hardware, operating environments, specialized frameworks and systems. There is an ongoing discussion on the need for an additional language, however one thing has become accepted - there is a need for a new strategy to cope with the development of integrated solutions.

Beyond the shift to a model-driven development with or without a new language, the proposed service orientation can provide a strategic approach to evolve to multi technology and multi-supplier integrated solutions, as for example demonstrated in practice by the new service-oriented toll management system developed by the Portuguese Brisa company [14]. The Brisa's experience however has shown that so far software development companies are not yet prepared to develop solutions under a cooperation approach. Furthermore, the pressure for rapid innovation does not give enough time to consolidate approaches for complex critical systems, where the risks are a strong inhibitor to the adoption of open software development patterns.

Therefore the proposed separation of contexts through collaboration layer, which was introduced above, is important, considering that not all organizations are expected to internally evolve to adopt a uniform open enterprise service bus (Figure 3). 


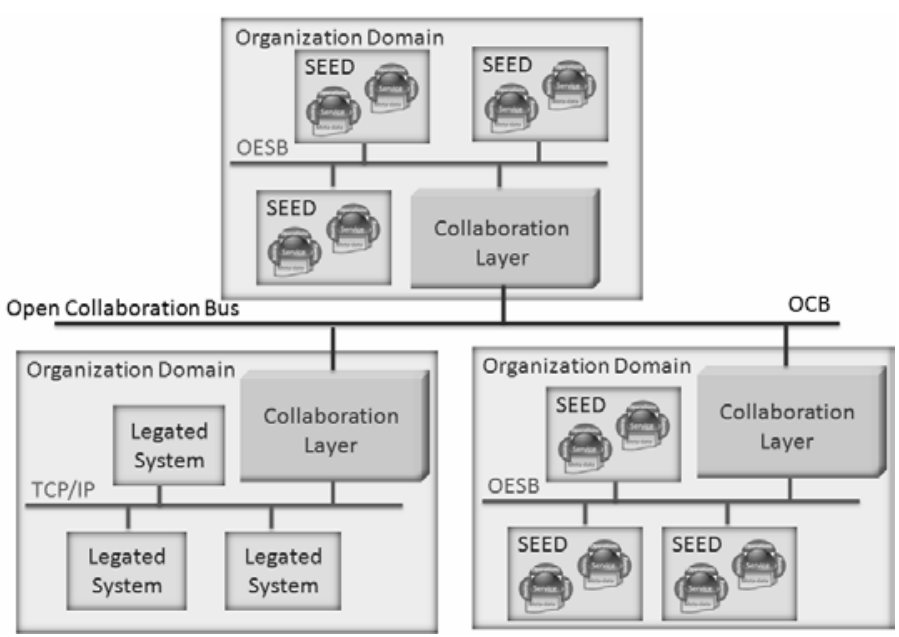

Figure 3. The Collaboration Layer abstracting internal network organization's architecture.

While such evolving strategy is important for an increasing reutilization of already existing computational resources, it is up to the collaboration layer to establish the required collaborative ecosystem, making it possible to offer the integrated business services. on one-to-one specific protocols; a service bus was not introduced yet as a strategy to get integrated solutions on an open basis (Figure 3 ). However the focus of this research is not the intra-enterprise information systems but rather its involvement in collaborative networks

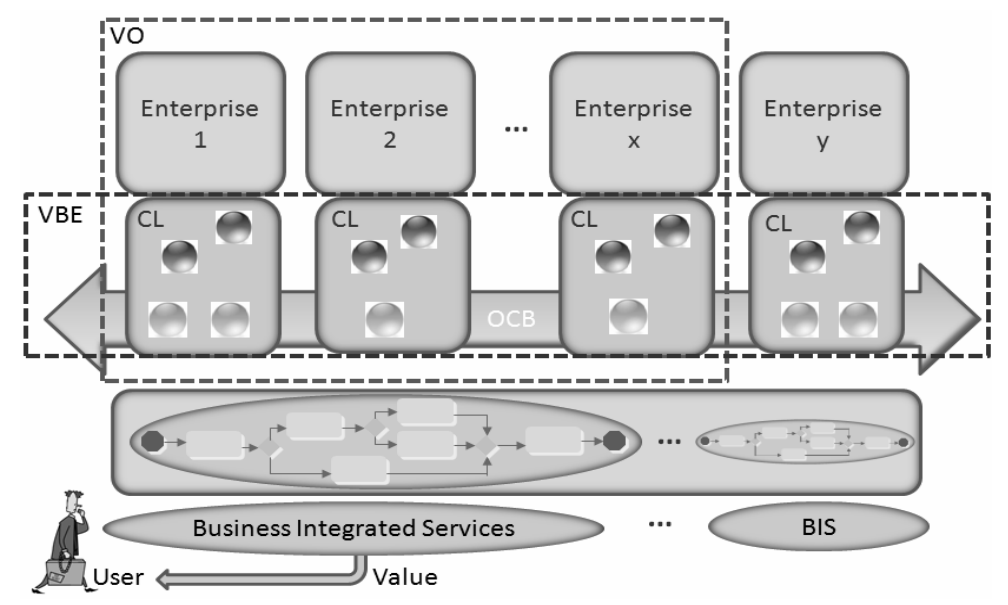

Figure 4. Business Integrated Services Framework Reference Architecture.

This above scenario suggests the assumption that an enterprise's information system might be at one of the following four possible stages: i) it presents a clear service oriented strategy considering it publishes the internal service through the OESB bus as a set of reusable resources (system and application services) to be considered by suppliers / developers of new services; ii) the enterprise is in the migration process and the enterprise service bus (OESB) only partially integrates its application systems, and thus through this bus it is not possible to access all the internal enterprise's systems; and iii) the enterprise is already organized in "islands of automation" while integrated applications are constructed
[1], [6] offering the integrated business services (Figure 4).

The proposed strategy illustrate in (Figure 4) above is to abstract the internal diversity of enterprises' information systems by considering only what is shared with others through the organizations' cooperation layer (CL). Therefore, the collaboration layer aims to play a proxy role, hiding the complexity of dealing with intra-enterprise systems and more important it is responsible to present a uniform view of each enterprise to all other participating organizations. It is clearly expected that a CL for a company that has not yet adopted the SOA approach is much more complex to develop than that for a company 
already owing an open service bus. For the SOA based company, the services in the CL might mainly address security issues and the management of resources needed to support the $\mathrm{CN}$. On the other hand, for the monolithic systems company, there is a need for the services to implement proprietary protocols and interfaces to make the necessary resources available through the CL to the collaborating community. The CL is also an important element for the realization of the open collaboration bus (OCB) concept, considering that all published systems and application services are implemented into the CL of the participating companies (Figure 4). In short, the collaboration layer (CL) of an enterprise is mainly responsible for the following:

i. It manages all enterprise resources that are shared within the virtual organization breeding environment (VBE) of which the enterprise is a member, and which can be employed through the life cycle of virtual organizations (VO). Availability of these resources through the CL is a part of each enterprise's participation preparedness;

ii. All services that an enterprise publishes in the OCB are implemented in its collaboration layer, even if a simple forwarding to the effective service provider inside the company is necessary. The CL implements both the system services and the application services. As stated above, the system services are part of a specific OCB signature while the application services do not follow so rigid rules considering that they are not part of the OCB.

The integrated services offered by a collaborative network establish a number of new challenges from the ICT viewpoint considering their intrinsic complexity. On one hand, there is a need for holistic approaches - one collaborative process might require the involvement of systems from different organizations - and, on the other hand, the important requirement to maintain solutions as much as possible independent from a specific supplier is an important precondition for the success of such collaborative businesses

\section{Related Work}

The definition of an ICT infrastructure able to support the complexity associated to the CNs is an open research topic. Research projects from diverse areas have attempted to tackle this problem but there is not yet a definite approach that can be considered agile and adaptive enough to receive a consensus as a reference strategy. The European integrated project ECOLEAD proposes that developing a plug-and-play horizontal ICT infrastructure (ICT-I) based on an open platformindependent specifications and ICT standards [16] can address this problem. With this approach potentially a collaborative business infrastructure $(\mathrm{CBI})$ can be derived as an open service oriented infrastructure to support the collaborative tasks of $\mathrm{CN}$ members in the context of collaborative processes. The proposed approach lacks however a clear strategy on how to cope with the complexity associated to the distribution and vulnerabilities (fault-tolerance, reliability, security, coordination) considering that organization's information system infrastructures follow different development and operation cultures. Our proposed collaboration layer aims to concentrate on the same reference architectural entity to resolve such diversities and embed the necessary coordination mechanisms, able to answer scalability and dependability requirements.

Other works more centered in the area of computer science and computer engineering, are focused on developing an open ICT infrastructure for the inter-organizational space, and can be exemplified by the case of NESSI, the European Technology Platform dedicated to Software and Services [12]. Another common strategy is to consider a centralized architecture as it can be exemplified by the case of the collaborative infrastructure for collaborative genetics experiences; the e-infrastructure to support collaborative embryo research [3]. The strategy might be acceptable for collaborative research and some other application domains but the integrated business service concept requires a federated approach considering that each organization holds its own culture, processes and technology, and thus the collaboration layer proposed in our research work is grounded on the participation of diverse independent and autonomous nodes. Strategic roadmaps on ICT and ageing as those being developed by the ePAL and 
BRAID projects [7] also require a new approach for service integration.

The discussed integrated business services in intelligent transport systems might involve large amount of stream (sensor) data collection, e.g. for real-time traffic analysis. The Infosphere Stream project/product from IBM is an example of a system addressing this challenge [4]. Nevertheless, a strategy is needed to make possible to plug such a specialized functionality in a complex open integrated solution. An open question is how to dynamically discover, assemble and deploy such a specialized subsystem considering that the integrated business service involves a network of organizations (stakeholders with their own IT infrastructure and systems).

\section{Conclusions}

This paper proposes a collaborative network approach to the development of the integrated business service concept. The development of integrated business services based on existing approaches results on complex closed IT systems with strong dependencies from its developer. The adoption of existing service oriented strategies is not a solution for the dynamics of the new collaborative business models. The research work is grounded on the acquired experience with already deployed business solutions requiring the participation of multiple stakeholders considering a single contract for the end users. The case of the Portuguese Via-Verde, initially developed for the tolling service and now extended to payments in parking areas and gas stations, provides a practical guiding background. Another important example is the provision of care services to elderly by multiple stakeholders.

One important aspect is the federative characteristic considering that organizations have their own process and technological culture what recalls for a strategy that guarantees their independence while committed to the collaborative network. In our proposed approach, it is up to the collaboration layer to support and implement mechanisms able to execute the collaborative processes underlying the business models associated to the offered integrated business services. The proposed collaboration layer is composed of cooperative services systems as autonomous computational entities with their associated meta-information able to dynamically adapt, as required for the development of collaborative business processes. One additional complexity addressed by our approach is the need for global coordination of the participating autonomous computational services which run on distributed and heterogeneous computational platforms. This second complexity is outside the scope of this paper and will be addressed in forthcoming papers. Furthermore, dependability considering security, reliability, fault tolerance, scalability from other quality issues, represents complex requirements that need to be addressed in the future and guaranteed in such federated collaborative network. The solution approach, must be balanced considering key issues from technology to process and the business levels, and requires a clear modeling framework and innovative mechanisms contributing to the development of collaborative solutions for collaborative network businesses.

\section{Acknowledgements}

This work was partially supported by BRISA Innovation and Technology company through a research and development project, and by the European Commission through the FP7 BRAID (grant agreement ICT-2009-7.1 2484852) project.

\section{REFERENCES}

1. AFSARMANESH, H., L. CAMARINHA-MATOS, A Framework for Management of Virtual Organization Breeding Environments, in Luis Camarinha-Matos, Hamideh Afsarmanesh, Angel Ortiz, eds., Collaborative Networks and Their Breeding Environments, vol. 186 of IFIP Intl. Fed. for Information Processing, Springer Boston, 2005, pp. 35-48, 10.1007/0-387-29360-4_4.

2. AFSARMANESH, H., L. M. CAMARINHA-MATOS, ERMILOVA, Vbe Reference Framework, in Luis M. CamarinhaMatos, Hamideh Afsarmanesh, Martin 
Ollus, eds, Methods and Tools for Collaborative Networked Organizations, Springer US, 2008, pp. 35-68, 10.1007/978-0-387-79424-2_2.

3. BARKER, A., J. I. van HEMERT, R. A. BALDOCK, M. P. ATKINSON, An eInfrastructure to Support Collaborative Embryo Research, in CCGRID '09: Proc. of the 2009 9th IEEE/ACM Intl. Sym. on Cluster Computing and the Grid, IEEE Computer Society, Washington, DC, USA, 2009, pp. 520-525.

4. BIEM, A., E. BOUILLET, H. FENG, A. RANGANATHAN, A. RIABOV, O. VERSCHEURE, H. KOUTSOPOULOS, C. MORAN, Ibm Infosphere Streams for Scalable, Real-time Intelligent Transportation Services, in Proc. of the 2010 Intl. Conf. on Management of Data, SIGMOD '10, ACM, New York, NY, USA, 2010, pp. 1093-1104.

5. CAMARINHA-MATOS, L. M., H. AFSARMANESH, M. OLLUS, Ecolead and Cno Base Concepts, in Luis $M$. Camarinha-Matos, Hamideh Afsarmanesh, Martin Ollus, eds, Methods and Tools for Collaborative Networked Organizations, Springer US, 2008, pp. 332. 10.1007/978-0-387-79424-2_1.

6. CAMARINHA-MATOS, L., H. AFSARMANESH, The Emerging Discipline of Collaborative Networks, in Luis Camarinha-Matos, ed., Virtual Enterprises and Collaborative Networks, vol. 149 of IFIP Intl. Fed. for Information Processing, Springer Boston, 2004, pp. 3-16, 10.1007/1-4020-8139-1_1.

7. CAMARINHA-MATOS, L., H. AFSARMANESH, The Need for a Strategic R\&D Roadmap for Active Ageing, in Leveraging Knowledge for Innovation in Collaborative Networks, vol. 307 of IFIP Advances in Information and Communication Technology, Springer Boston, 2009, pp. 669-681, 10.1007/978-3-642-04568-4_69.

8. ENGELS. G., M. ASSMANN, ServiceOriented Enterprise Architectures: Evolution of Concepts and Methods, in Enterprise Distributed Object Computing Conf., 2008. EDOC '08. 12th Intl. IEEE, 2008, pp. xxxiv -xliii.
9. LASKEY, K., et al. Reference Architecture Foundation for Service Oriented Architecture Version 1.0. OASIS - Service Oriented Architecture Reference Model TC, October 2009.

10. MARGOLIS, B., IBM Rational Business Developer with EGL. Mc Press, 2008.

11. NIKOLAO, C., M. BITSAKI, Towards a Theory of Emergent Service Value Networks - and an Application, in An e-Learning Service Economy in FET Proactive Workshop on Objective IST2007.8.4. The Science of Complex Systems for Socially Intelligent ICT Complexity Research Projects Forum, ECCS’07 Dresden, October 2007.

12. NIKOLOV, R., S. ILIEVA, Building a Research University Ecosystem: the Case of Software Engineering Education at Sofia University, in ESEC-FSE companion '07: The 6th Joint Meeting on European Software Engineering Conf. and the ACM SIGSOFT, ACM, New York, NY, USA, 2007, pp. 491-500.

13. OSÓRIO, A., H. AFSARMANESH, L. CAMARINHA-MATOS, Towards a Reference Architecture for a Collaborative Intelligent Transport System Infrastructure, in Collaborative Networks for a Sustainable World, vol. 336 of IFIP Advances in Information and Communication Technology, Springer Boston, 2010, pp. 469-477, 10.1007/9783-642-15961-9_56.

14. OSÓRIO, A., L. CAMARINHAMATOS, Towards a Distributed Process Execution Platform for Collaborative Networks, in, Information Technology for Balanced Manufacturing Systems, vol. 220 of IFIP, Springer Boston, 2006, pp. 233-240, 10.1007/9780-387-36594-7_25.

15. OSÓRIO. A., L. CAMARINHAMATOS, J. GOMES, A Collaborative Network Case Study: The Extended "Via-verde" Toll Payment System, in Collaborative Networks and Their Breeding Environments, vol. 186 of IFIP International Federation for Information 
Processing, Springer Boston, 2005, pp. 559-568, 10.1007/0-387-29360-4_59.

16. RABELO, R. J., Advanced Collaborative Business ICT Infrastructures, in Methods and Tools for Collaborative Networked Organizations, Springer US, 2008, pp. 337-370, 10.1007/978-0-387-79424-2_14.

17. ROLLAND, C., S. NURCAN, G. GROSZ, A Unified Framework for Modelling Cooperative Design Processes and Cooperative Business Processes, in System Sciences, 1998, Proc. of the Thirty-First Hawaii International Conference, vol. 5, January 1998, pp. 376-385.

18. STEPHAN, R., J. MÜLLER, B. BAUER, An Evaluation and Decision Method for ICT Architectures for Crossorganizational Business Process Coordination, Information Systems and E-Business Management, 2010, pp. 1-38, 10.1007/s10257-010-0141-5.

19. SANDERS, D. T., J. A. HAMILTON JR., R. A. MACDONALD, Supporting a Service-oriented Architecture, in Proc. of the 2008 Spring simulation multiconference, SpringSim '08, Society for Computer Simulation International, San Diego, CA, USA, 2008, pp. 325-334.
20. SKOPIK, F., D. SCHALL, S. DUSTDAR, Trusted Interaction Patterns in Large-scale Enterprise Service Networks, February 2010, pp. $367-374$.

21. TRONCOSO, C., G. DANEZIS, E. KOSTA, B. PRENEEL, Pripayd: Privacy Friendly Pay-as-you-drive Insurance, in WPES '07: Proceedings of the 2007 ACM workshop on Privacy in electronic society, ACM, New York, NY, USA, 2007, pp. 99-107.

22. ZHANG, L.-J., Eic editorial: Introduction to the Knowledge Areas of Services Computing, IEEE Transactions on Services Computing, vol. 1, 2008, pp. 62-74.

23. SAAD, S., H. ZGAYA, S. HAMMADI, Novel Ontology Model for Communicating Heterogeneous Negotiation Mobile-Agent in a Transport Environment, Studies in Informatics and Control Journal, Vol. 17, No. 4/2008, pp. 333-352. 